\title{
A mutational profile in multiple thymic squamous cell carcinoma
}

\author{
Chun Jin ${ }^{1 \#}$, Cheng Yan ${ }^{2 \#}$, Yi Zhang ${ }^{1 \#}$, Yong-Xing Zhang ${ }^{1}$, Jia-Hao Jiang ${ }^{1}$, Jian-Yong Ding ${ }^{1}$ \\ ${ }^{1}$ Department of Thoracic Surgery, ${ }^{2}$ Department of Radiology, Zhongshan Hospital, Fudan University, Shanghai 200032, China \\ Contributions: (I) Conception and design: C Jin, JH Jiang, JY Ding; (II) Administrative support: JY Ding; (III) Provision of study materials or patients: \\ C Yan, Y Zhang, YX Zhang; (IV) Collection and assembly of data: C Jin, JH Jiang; (V) Data analysis and interpretation: JH Jiang, JY Ding; (VI) \\ Manuscript writing: All authors; (VII) Final approval of manuscript: All authors. \\ \#These authors contributed equally to this work. \\ Correspondence to: Jia-Hao Jiang. Department of Thoracic Surgery, Zhongshan Hospital, 180 Fenglin Road, Shanghai 200032, China. \\ Email: jiang.jiahao@zs-hospital.sh.cn.
}

\begin{abstract}
Background: Multiple thymic squamous cell carcinoma (TSCC) is a rare thymic epithelial tumor with a dismal prognosis. Mutational profiles of multiple TSCC may expand our understanding of tumorigenesis and treatment options for these tumors.

Methods: We sequenced the whole exomes of 3 TSCC nodules from a multiple TSCC patient and a paired peripheral blood sample and identified single-nucleotide variants and small insertions and deletions, and also performed gene ontological and pathway analyses.

Results: The 3 TSCC nodules were subjected to hematoxylin-eosin staining, and the results showed that these 3 nodules were highly similar with respect to histology. We identified 116, 94 and 98 non-synonymous somatic mutations in the 3 TSCC nodules, and 34 mutations, including mutations in TP53 and ARID1A, among others, were present in all 3 TSCC nodules. We then performed immunohistochemistry to assess two selected genes, TP53 and ARID1A, and found that the 3 TSCC nodules expressed similar levels of TP53 and $A R I D 1 A$. Further gene ontological analysis and pathway analysis revealed that the 3 TSCC nodules also had similar significantly enriched pathways based on the identified genetic alterations. These results demonstrated that the 3 multiple TSCC nodules were spatially independent of each other but were highly similar with respect to histological sources and genetic characteristics, suggesting that 2 TSCC nodules were likely metastases of the third nodule.
\end{abstract}

Conclusions: These findings suggest that TSCC cells can be transferred to other sites inside the thymus and that total thymectomy is a good treatment option for thymic epithelial tumors.

Keywords: Thymic squamous cell carcinoma (TSCC); exome sequencing; thymectomy

Submitted Aug 07, 2019. Accepted for publication Nov 04, 2019.

doi: 10.21037 /gs.2019.11.08

View this article at: http://dx.doi.org/10.21037/gs.2019.11.08

\section{Introduction}

Thymoma and thymic carcinoma are rare neoplasms that are nevertheless the most common tumors of the mediastinum. Thymic carcinomas exhibit more aggressive properties and higher metastatic potential than thymoma (1). Thymic squamous cell carcinoma (TSCC), which is the most common type of thymic carcinoma, is not particularly responsive to chemotherapy or radiotherapy. Radical resection is the primary treatment option and possibly the only potentially curative option for TSCC.

The thymic lobules have a capsule and the mediastinal fat is confined by pleura. When thymic tumors are accompanied by myasthenia gravis, total thymectomy is required to remove the thymus, all adipose tissue in the anterior mediastinum between the two phrenic nerves, and the left and right cardiophrenic angle lymph nodes (2). Tumor recurrence may occur if the TSCC and surrounding adipose tissue are not completely removed. The carcinogenesis of TSCC is unclear, and the manifestations 

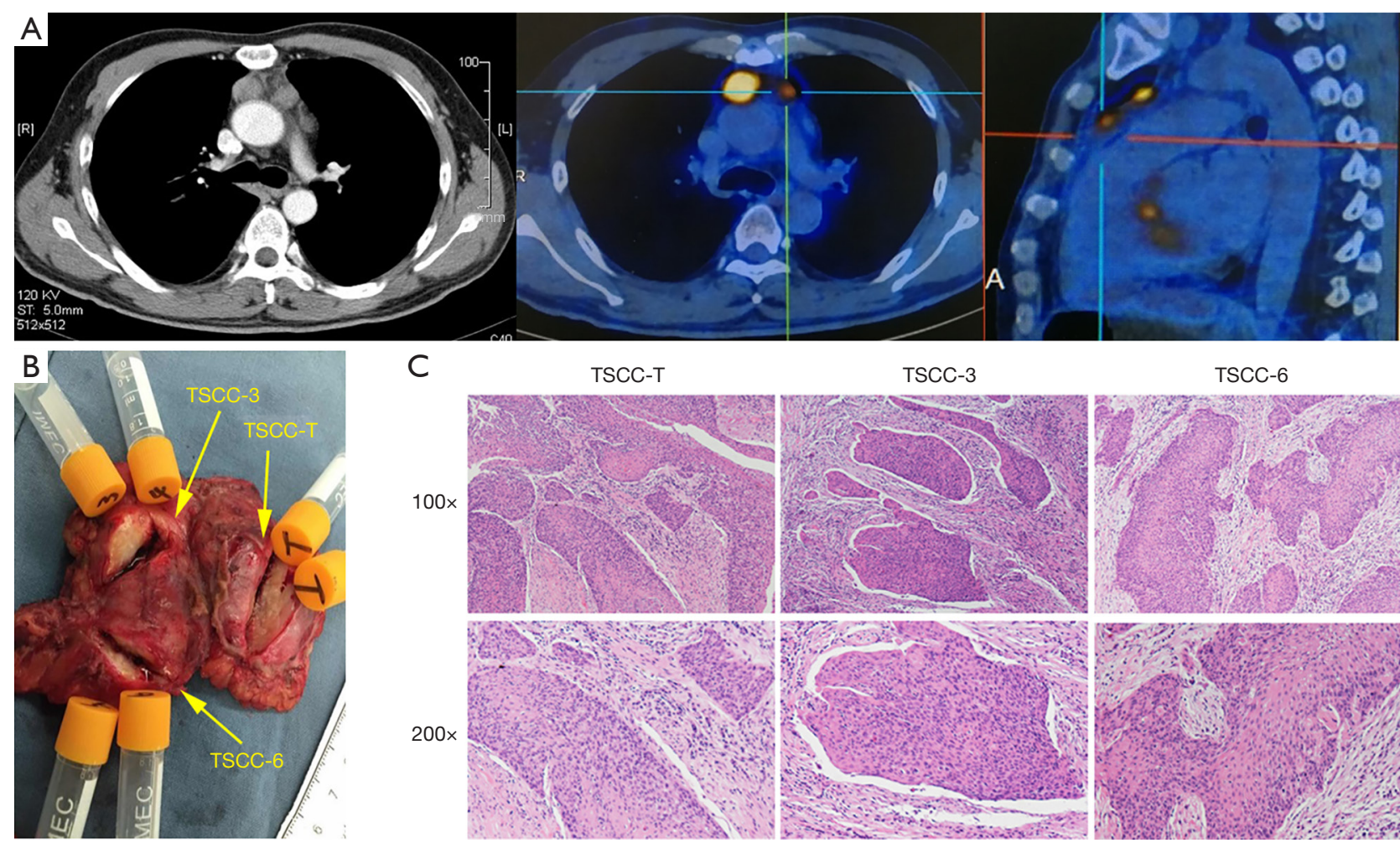

C
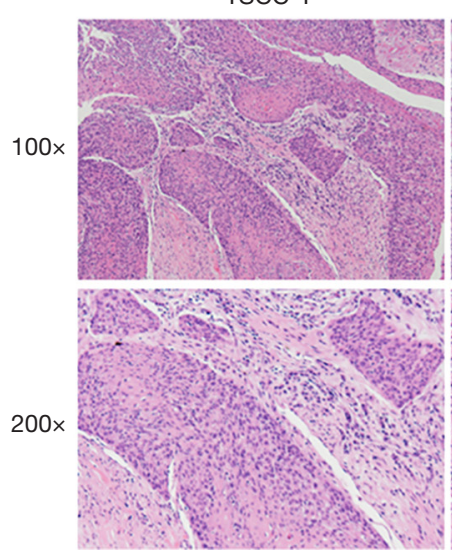

TSCC-3

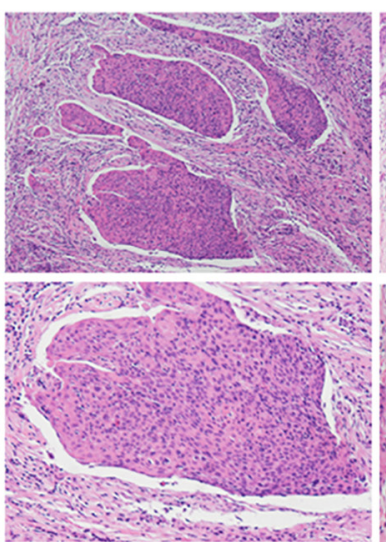

TSCC- 6

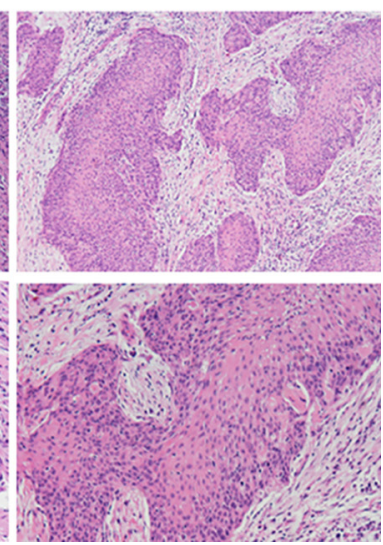

Figure 1 Clinical information for the patient. (A) Representative CT and PET/CT images; (B) removal of thymic squamous cell carcinoma (TSCC) nodules; (C) hematoxylin-eosin staining of 3 thymic nodules.

of this disease vary. Most TSCC are single, and multiple TSCC is extremely uncommon. Multiple TSCC can present as multiple primary tumors or as metastatic nodules. Each nodule in multiple TSCC harbors unique biological information that can enrich our knowledge regarding $\operatorname{TSCC}(3,4)$.

In this study, we sequenced the whole exomes of 3 TSCC nodules in a multiple TSCC patient and a paired peripheral blood sample and identified single-nucleotide variants and small insertions and deletions. We sought to elucidate the mutational profiles of multiple TSCC and expand our understanding of tumorigenesis and treatment options for these tumors.

\section{Methods}

\section{Patients and samples}

A thymic mass was incidentally found in a 45-year-old man 10 days before his admission to the hospital. Positron emission tomography (PET)/computed tomography (CT) indicated that the patient had three thymic nodules with diameters of 3.0, 1.5 and $1.2 \mathrm{~cm}$ and max standard uptake values (SUVmax) of 12.2, 8.1 and 8.1, respectively. He underwent extended thymectomy for multiple thymic neoplasms at Zhongshan Hospital, Fudan University (Shanghai, China) and was pathologically diagnosed with multiple non-keratinizing squamous cell carcinomas (Figure 1). We obtained a peripheral blood sample and three tumor tissue samples from this patient (Figure S1). Genomic DNA samples were extracted from freshly frozen tumor tissues and mononuclear cells (isolated from peripheral blood via Ficoll gradient centrifugation) from this TSCC patient for whole-exome sequencing. Written informed consent was obtained from the patient, and ethical approval was obtained from the Zhongshan Hospital Research Ethics Committee.

\section{Whole-exome capture sequencing and bioinformatics analysis}

Previously, described whole-exome capture sequencing methods were used (5). Candidate somatic mutations were 


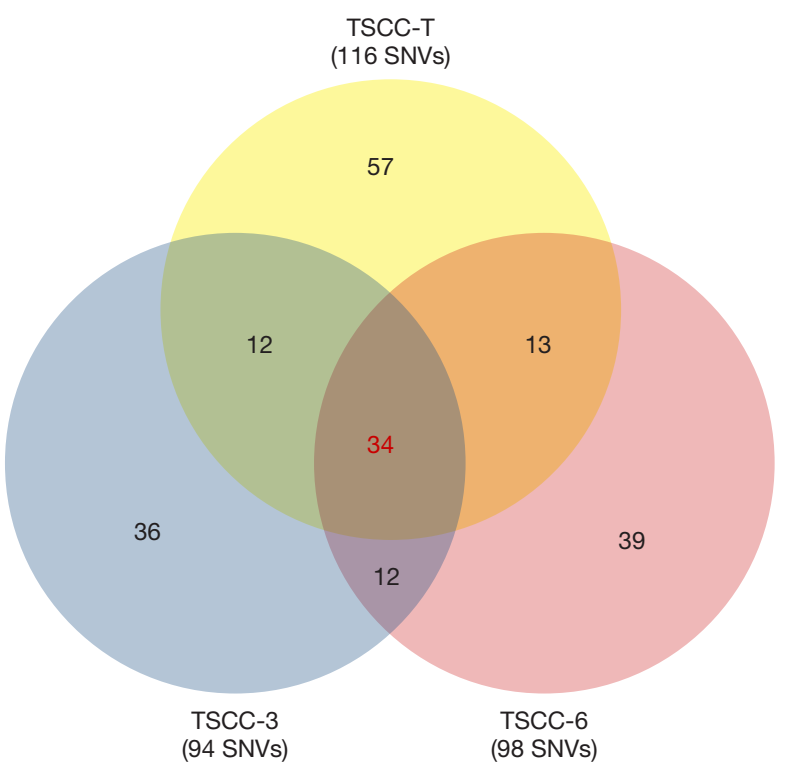

Figure 2 Identified gene mutations of multiple thymic squamous cell carcinoma (TSCC) nodules. SNV, single-nucleotide variations.

identified using a bioinformatics pipeline, as described in Figure $S 2$.

\section{Immunobistochemistry}

Hematoxylin-eosin staining and immunohistochemistry were performed as previously described (6). Specific primary antibodies against TP53 and ARID1A (Cell Signaling Technology, Beverly, MA, USA) were used.

\section{Results}

\section{Mutational profiles of multiple TSCC}

To obtain insights into genetic alterations that characterize TSCC, we examined a TSCC patient with multiple tumors and performed whole-exome sequencing analysis on 3 tumors and a matched peripheral blood sample. We identified 116, 94 and 98 somatic non-synonymous single-nucleotide variations (SNVs) and insertions/ deletions (indels) in coding regions in the 3 assessed TSCC nodules (Figure 2). To determine whether the multiple thymic nodules were derived from one single nodule with intrathymic metastases or multiple primary thymic tumors, we analyzed the distribution of all of the somatic mutations and observed that 71 somatic mutations were present in at least one tumor region and that 34 mutations, including mutations in TP53, ARID1A, and NOTCH1, among others, were present in all 3 TSCC nodules (Table 1). Moreover, without exception, these 34 mutations involved the same single nucleotide change at the same position in all 3 TSCC nodules. In prior studies, 409 cancerrelated genes were sequenced in 12 TSCC tissues with no recurrent mutations observed among the sequenced samples, indicating that TSCC is highly heterogeneous (7). Therefore, our results suggest that the multiple thymic nodules within the examined individual were likely derived from a single nodule.

To further understand the function of the identified gene mutations, we reviewed literature reports addressing these mutations and found that at present, most of these mutations have not been reported to be related to human tumors. Research has indicated that certain identified gene mutations, including mutations in TP53, ARID1A, NOTCH1, GNAQ, KMT2C and SPEN, play roles in human tumors, including thymic epithelial tumors (8-11).

\section{Identification of copy number variations}

Subsequently, to study TSCC-related gene amplification and loss, we detected copy number variations (CNVs) in samples from the examined patient and identified 393 CNVs involving 259 genes in all TSCC samples (Figure 3). Moreover, $58 \mathrm{CNVs}$ (involving the gain or loss of 37 genes), which accounted for approximately $15 \%$ of all identified CNVs, were common to all TSCC samples. Certain CNVs, including CNVs in SPTA1, PRKAR1A and $R A N B P 2$, have been reported to be related to human tumors and were first found to be related to human thymic epithelial tumors $(3,12)$.

\section{Analyses of SNV-enriched patbways}

Gene ontological and pathway analyses were also performed. These analyses revealed that the 3 TSCC nodules had similar significantly enriched pathways based on the identified genetic alterations. Certain pathways, including the GnRH, Notch and MAPK signaling pathways, may play a role in the development of TSCC (Figure 4).

\section{Histological similarity}

A preoperative enhanced chest CT scan indicated that this patient had 3 thymic nodules that were spatially independent of each other. All of these thymic nodules 
Table 1 Somatic recurrent mutations in all the 3 thymic squamous cell carcinoma (TSCC) nodules

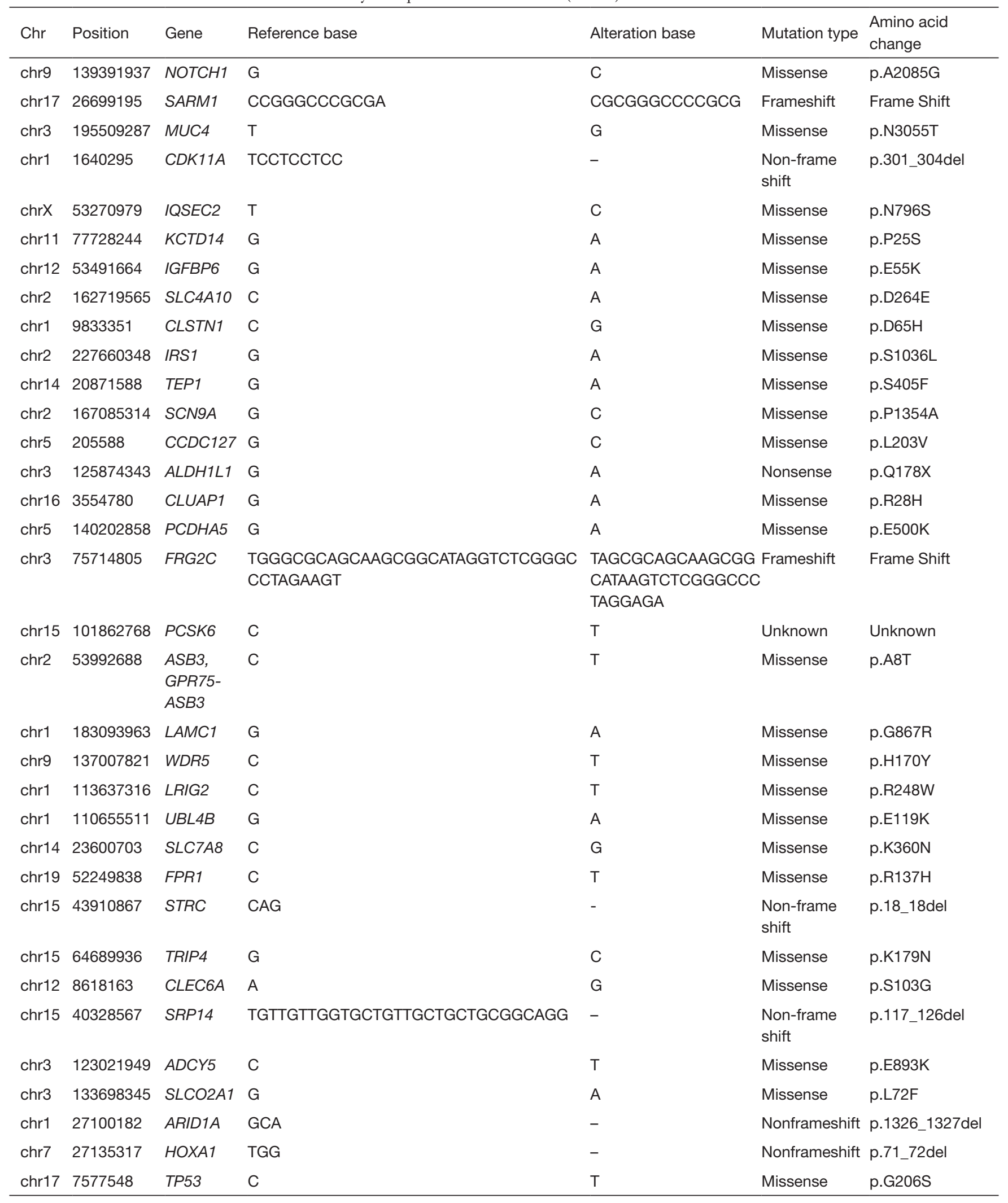



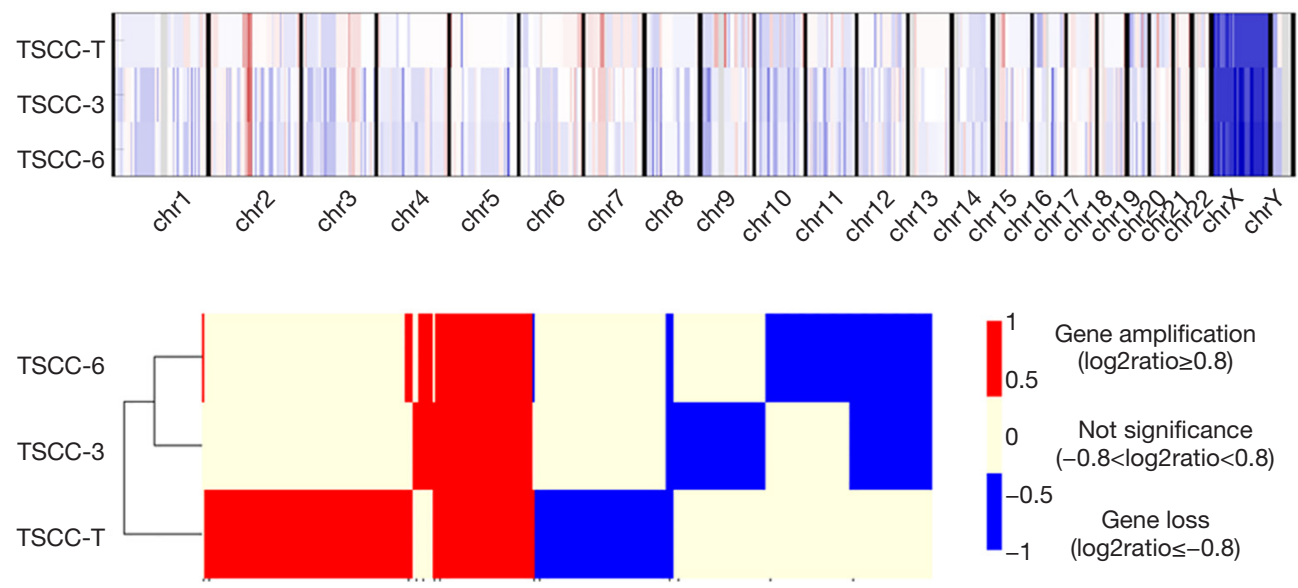

Figure 3 Copy number variations (CNVs) of multiple thymic squamous cell carcinoma (TSCC) nodules.

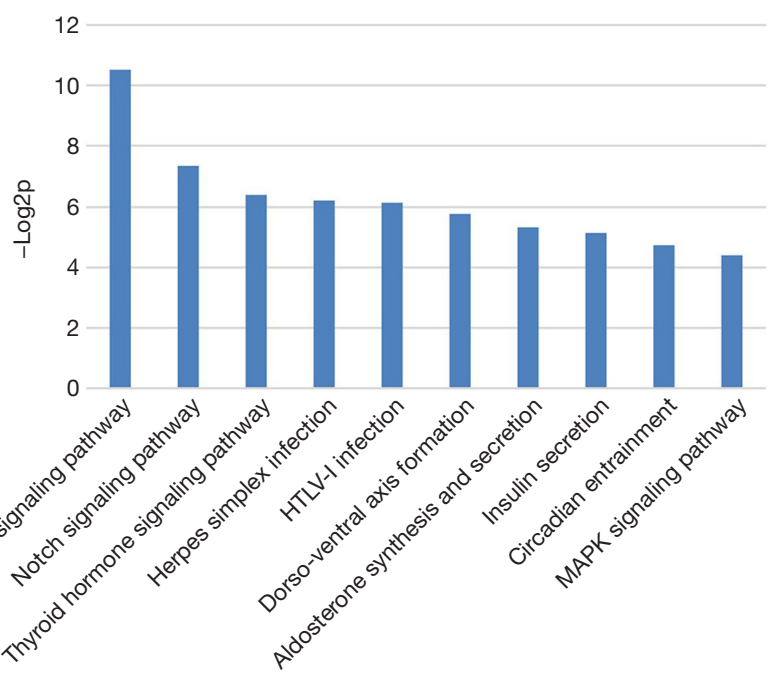

Figure 4 Enriched pathway analysis based on the identified genetic alterations.

were resected and pathologically diagnosed as squamous cell carcinoma. To analyze the histological sources of the 3 TSCC nodules, the nodules were subjected to hematoxylineosin staining, and pathologists concluded that all 3 nodules were highly similar with respect to histology and appeared to be derived from the same tumor. To further clarify the histological relationship among these 3 nodules, we performed immunohistochemistry to examine 2 mutated genes, TP53 and ARID1A, in these nodules and found that all 3 TSCC nodules expressed similar levels of ARID1A. TP53 expression was inhomogeneous in local tumor tissues but similar in overall tumor tissues (Figure 5).

\section{Discussion}

The development of thymic tumors involves dynamic interplay between thymic epithelial cells and their surrounding microenvironment $(7,13)$. Even within the same thymus, different thymic epithelial cells can evolve into diverse thymic epithelial tumors after experiencing a series of common or different genetic mutations. These different tumors are typically regarded as multicentric thymic tumors based on tumor heterogeneity $(14,15)$. However, in the present study, various genetic and histological data are presented to demonstrate the phenomenon that multiple thymic tumors may be derived from the same tumor clone, indicating that in multiple TSCC, certain thymic tumors are likely to be metastases from a primary tumor. These findings help to deepen our understanding of thymic epithelial tumors. The thymic lobules have a capsule which consists of thymus tissue, adipose tissue degenerated from the thymus after adulthood, and the thymus lymph nodes. Thus, the potential correlation between the primary tumor and the metastases reminds us that probable communication channels may present in the thymic lobules that facilitates tumor recurrence and metastasis. This communication channel may be present in both TSCC and thymoma. This finding suggests that total thymectomy is necessary for either thymic carcinoma or thymoma to reduce the likelihood of tumor recurrence and improve survival.

In addition, we observed that although the different TSCC nodules were extremely similar histologically, they did not exhibit identical genetic changes. In contrast, large differences were observed. Each tumor nodule had its own 


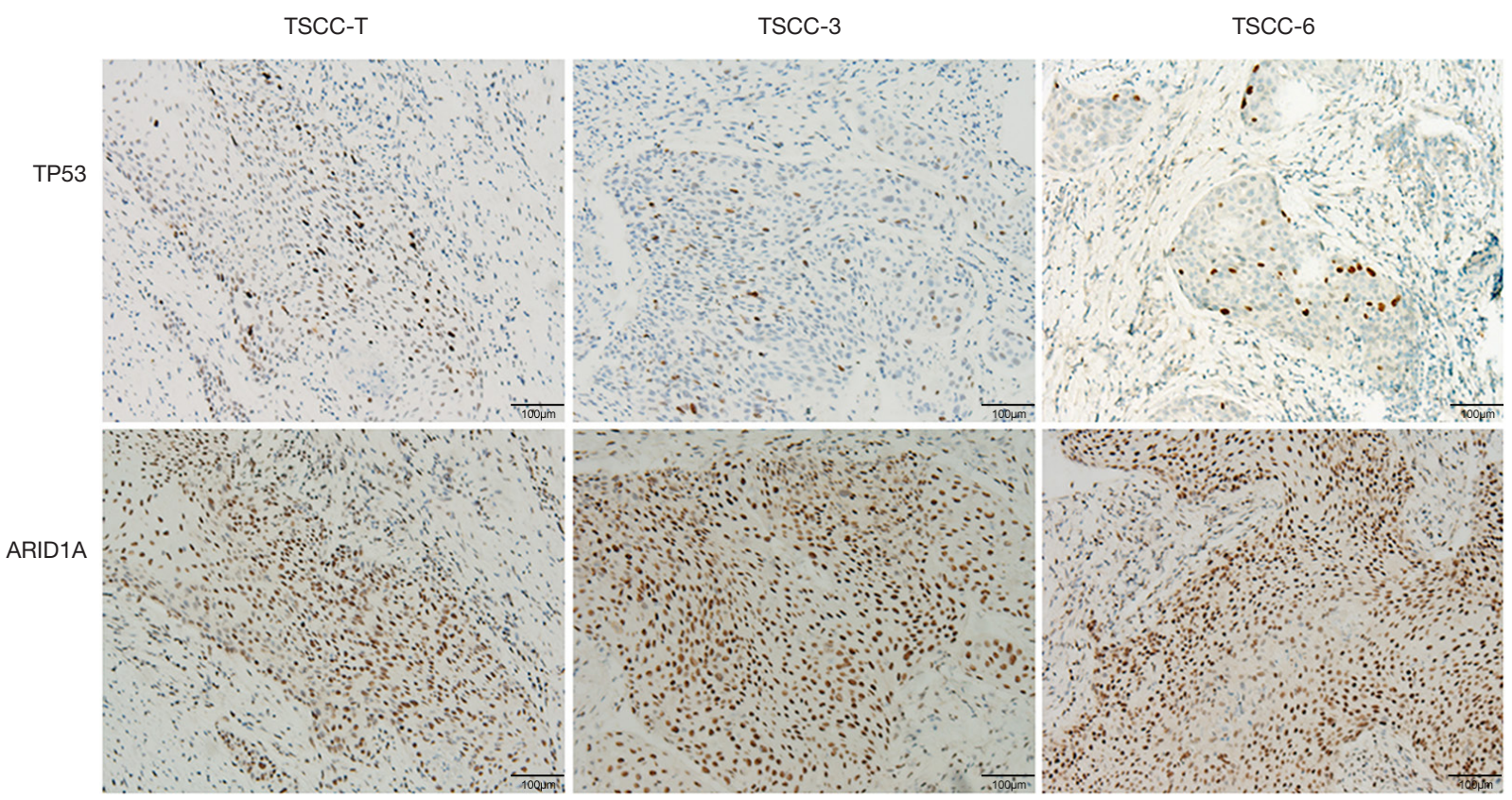

Figure 5 Immunohistochemistry of TP53 and ARID1A in multiple thymic squamous cell carcinoma (TSCC) nodules.

unique genetic changes that distinguished it from the other tumor nodules. These findings suggest that metastatic TSCC tumors continue to undergo evolutionary processes and gradually become new TSCC nodules that differ from primary tumors. This implication may help us understand the mechanisms of recurrence, metastasis and drug resistance in TSCC.

While the genetics of thymic carcinomas has been reported, few has focused on the genetic alternations in the multiple thymic carcinoma given to its rarity. Thus, the exome sequencing of multiple TSCC provides the complement to the profile of genetic aberrations in thymic epithelial carcinoma. We also detected genetic aberrations, including mutations in TP53, ARID1A, NOTCH1, GNAQ, KMT2C and SPEN, and CNVs, including CNVs in SPTA1, PRKAR1A, and RANBP2, that are likely to be associated with TSCC. In prior reports, exome sequencing showed that TP53 was the most frequently mutated gene in TSCC and that TP53 mutation was associated with a higher rate of recurrence $(8,16)$. Other studies have demonstrated that the disruption of NOTCH1 pathways is involved in the development of thymic mucoepidermoid carcinoma (9). Thus, some genetic aberrations may ubiquitously occur in thymic epithelial tumors. However, the functions of most of the identified genes associated with thymic epithelial tumors remain unclear. These genes may be involved in tumor development and progression in unknown ways. Further research is needed to clarify these issues.

In conclusion, our findings suggest that there may be a potential communication channel in the thymic capsule that likely facilitates thymic tumor recurrence and metastasis. Thus, total thymectomy may be a good treatment option for not only malignant thymic epithelial tumor patients but also thymoma patients.

\section{Acknowledgments}

None.

\section{Footnote}

Conflicts of Interest: The authors have no conflicts of interest to declare.

Ethical Statement: The authors are accountable for all aspects of the work in ensuring that questions related to the accuracy or integrity of any part of the work are appropriately investigated and resolved. Written informed consent was obtained from the patient, and ethical approval was obtained from the Zhongshan Hospital Research Ethics Committee. 


\section{References}

1. Thomas A, Rajan A, Berman A, et al. Sunitinib in patients with chemotherapy-refractory thymoma and thymic carcinoma: an open-label phase 2 trial. Lancet Oncol 2015;16:177-86.

2. Di Crescenzo VG, Napolitano F, Panico C, et al. Surgical approach in thymectomy: Our experience and review of the literature. Int J Surg Case Rep 2017;39:19-24.

3. Lee Y, Park S, Lee SH, Lee H. Characterization of genetic aberrations in a single case of metastatic thymic adenocarcinoma. BMC Cancer 2017;17:330.

4. Girard N, Shen R, Guo T, et al. Comprehensive genomic analysis reveals clinically relevant molecular distinctions between thymic carcinomas and thymomas. Clin Cancer Res. 2009;15:6790-9.

5. Jiang JH, Liu YF, Ke AW, et al. Clinical significance of the ubiquitin ligase $\mathrm{UBE} 3 \mathrm{C}$ in hepatocellular carcinoma revealed by exome sequencing. Hepatology 2014;59:2216-27.

6. Ke AW, Shi GM, Zhou J, et al. CD151 Amplifies Signaling by Integrin alpha6beta 1 to PI3K and Induces the Epithelial-Mesenchymal Transition in HCC Cells. Gastroenterology 2011;140:1629-41.e1615.

7. Shitara M, Okuda K, Suzuki A, et al. Genetic profiling of thymic carcinoma using targeted next-generation sequencing. Lung Cancer 2014;86:174-9.

8. Moreira AL, Won HH, McMillan R, et al. Massively parallel sequencing identifies recurrent mutations in TP53 in thymic carcinoma associated with poor prognosis.

Cite this article as: Jin C, Yan C, Zhang Y, Zhang YX, Jiang JH, Ding JY. A mutational profile in multiple thymic squamous cell carcinoma. Gland Surg 2019;8(6):691-697. doi: 10.21037/ gs.2019.11.08
Journal of thoracic oncology : official publication of the International Association for the Study of Lung Cancer. 2015;10:373-80.

9. Prieto-Granada CN, Inagaki H, Mueller J. Thymic Mucoepidermoid Carcinoma: Report of a Case With CTRC1/3-MALM2 Molecular Studies. Int J Surg Pathol 2015;23:277-83.

10. Wang K, Kan J, Yuen ST, et al. Exome sequencing identifies frequent mutation of ARID1A in molecular subtypes of gastric cancer. Nat Genet 2011;43:1219-23.

11. Asao T, Fujiwara Y, Sunami K, et al. Medical treatment involving investigational drugs and genetic profile of thymic carcinoma. Lung Cancer 2016;93:77-81.

12. Kim DH, Kwon S, Byun S, et al. Critical role of RanBP2-mediated SUMOylation of Small Heterodimer Partner in maintaining bile acid homeostasis. Nature Communications 2016;7:12179.

13. Mokhtar M, Kondo K, Namura T, et al. Methylation and expression profiles of MGMT gene in thymic epithelial tumors. Lung Cancer 2014;83:279-87.

14. Yoneda S, Matsuzoe D, Kawakami T, et al. Synchronous multicentric thymona: report of a case. Surg Today 2004;34:597-9.

15. Ishibashi H, Akamatsu H, Sunamori M. Multiple thymoma with myasthenia gravis: report of a case. Surg Today 2003;33:49-51.

16. Hu Z, Wang J, Yao T, et al. Identification of novel mutations of TP53, ALK and RET gene in metastatic thymic squamous cell carcinoma and its therapeutic implication. Lung Cancer 2013;81:27-31. 


\begin{tabular}{|c|c|c|c|c|}
\hline & Normal & TSCC-T & TSCC-3 & TSCC-6 \\
\hline chr1_65311116 & 2 & 2 & 2 & 2 \\
\hline chr1_158607935 & 1 & 1 & 1 & 1 \\
\hline chr1_158612236 & 2 & 2 & 2 & 2 \\
\hline chr1_204403659 & 1 & 1 & 1 & 1 \\
\hline chr1_204515863 & 0 & 0 & 0 & 0 \\
\hline chr11_60230722 & 2 & 2 & 2 & 2 \\
\hline chr11_125497466 & 1 & 1 & 1 & 1 \\
\hline chr12_57579727 & 0 & 0 & 0 & 0 \\
\hline chr12_92539378 & 0 & 0 & 0 & 0 \\
\hline chr14_105246325 & 0 & 0 & 0 & 0 \\
\hline chr15_67476970 & 0 & 0 & 0 & 0 \\
\hline chr15_75091247 & 1 & 1 & 1 & 1 \\
\hline chr15_90634941 & 0 & 0 & 0 & 0 \\
\hline chr16_359953 & 0 & 0 & 0 & 0 \\
\hline chr16_81971403 & 0 & 0 & 0 & 0 \\
\hline chr17_8108339 & 1 & 1 & 1 & 1 \\
\hline chr17_41215825 & 0 & 0 & 0 & 0 \\
\hline chr17_47696820 & 1 & 1 & 1 & 1 \\
\hline chr17_70120551 & 1 & 1 & 1 & 1 \\
\hline chr17_78681590 & 2 & 2 & 2 & 2 \\
\hline chr19_10599965 & 1 & 1 & 1 & 1 \\
\hline chr19_42793590 & 1 & 1 & 1 & 1 \\
\hline chr19_50909389 & 0 & 0 & 0 & 0 \\
\hline chr2_141762830 & 2 & 2 & 2 & 2 \\
\hline chr2_198265526 & 2 & 2 & 2 & 2 \\
\hline chr21_36421036 & 0 & 0 & 0 & 0 \\
\hline chr3_47079112 & 2 & 2 & 2 & 2 \\
\hline chr4_55125058 & 1 & 1 & 1 & 1 \\
\hline chr5_35857235 & 1 & 1 & 1 & 1 \\
\hline chr5_56183941 & 2 & 2 & 2 & 2 \\
\hline chr5_67569746 & 0 & 0 & 0 & 0 \\
\hline chr5_149516480 & 1 & 1 & 1 & 1 \\
\hline chr5_176720766 & 0 & 0 & 0 & 0 \\
\hline chr6_397290 & 1 & 1 & 1 & 1 \\
\hline chr6_41903782 & 1 & 1 & 1 & 1 \\
\hline chr6_43746169 & 1 & 1 & 1 & 1 \\
\hline chr7_55214348 & 1 & 1 & 1 & 1 \\
\hline chr7_141420768 & 1 & 1 & 1 & 1 \\
\hline chr8_37696874 & 2 & 2 & 2 & 2 \\
\hline chr8_69143520 & 1 & 1 & 1 & 1 \\
\hline chr8_139263328 & 0 & 0 & 0 & 0 \\
\hline
\end{tabular}

Figure $\mathbf{S 1}$ The peripheral blood sample and three tumor tissue samples from one same patient. 


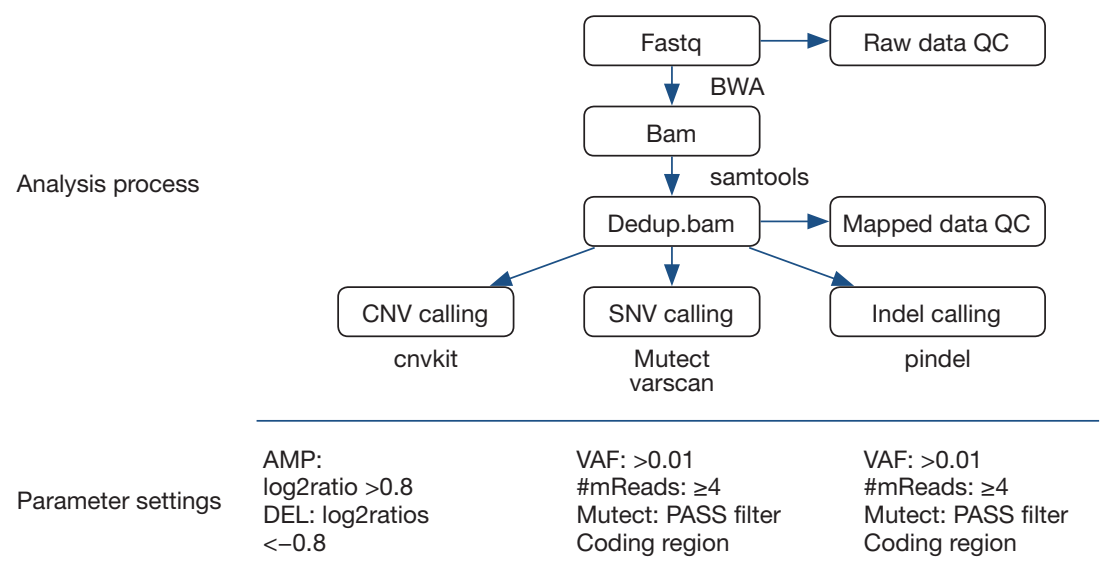

Figure S2 The bioinformatics pipeline of candidate somatic mutations. 